Supporting Information

\title{
Metal Nanoparticles Confined in the Nanospace of Double-shelled Hollow Silica Spheres for Highly Efficient and Selective Catalysis
}

\author{
Shunsheng Cao ${ }^{a, b}$ Jun Chang, ${ }^{a}$ Long Fang, ${ }^{a}$ Limin $W u{ }^{* b}$
}

\begin{abstract}
${ }^{\mathrm{a}}$ School of Materials Science and Engineering, Jiangsu University, Zhenjiang 212013, China;
${ }^{\mathrm{b}}$ Department of Materials Science and State Key Laboratory of Molecular Engineering of Polymers, Fudan University, Shanghai 200433, China.

*E-mail: 1mw@fudan.edu.cn
\end{abstract}

\section{Experimental Section}

Materials. Unless otherwise noted, chemicals were available from J \& K scientific Ltd and used as received. Styrene, $\mathrm{K}_{2} \mathrm{~S}_{2} \mathrm{O}_{8}$, sodium borohydride, ammonia, and absolute ethanol were the products of the Sinopharm Chemical Reagent Co., Ltd. (China) and were used as received except styrene was purified with 5 wt.\% $\mathrm{NaOH}$ solution prior to use. Deionized water was collected from the Direct-Q UV System (Millipore).

Immobilization of silver ions. The monodisperse polystyrene spheres (PS)/ $\mathrm{SiO}_{2}$ core-shell colloidal spheres were synthesized according to our previous method, ${ }^{\mathrm{S} 1}$ and further coated by using the impregnation method as follows: ${ }^{\mathrm{S} 2}$ Typically, $\mathrm{PS} / \mathrm{SiO}_{2}$ spheres $(4 \mathrm{~g})$ were added to $40 \mathrm{~mL}$ of aqueous solution containing the required amount of metal precursor salts $\left(0.05 \mathrm{~g} \mathrm{AgNO}_{3}\right)$, and stirred at $50{ }^{\circ} \mathrm{C}$ for $24 \mathrm{~h}$, evaporated and dried under vacuum overnight.

Preparation of Ag-DHSS. The synthesized PS@SiO $\mathrm{S}_{2} @ \mathrm{Ag}^{+}$hybrid colloidal spheres were further in situ polymerized with styrene and co-monomer (2-(methacryloyloxy) ethyltrimethylammonium chloride) at $70^{\circ} \mathrm{C}$ for $24 \mathrm{~h}$ to obtain sandwich-like PS@SiO ${ }_{2} @ \mathrm{PS}$ colloidal spheres. These particles 
were further treated by using TEOS under the ammonia as above to generate the exterior silica layer. After calcination at $450^{\circ} \mathrm{C}$ and then reduction by sodium borohydride, Ag-DHSS were obtained.

Characterization. The internal and external morphologies of the DHSS and Ag-DHSS were characterized using transmission electron microscopy (TEM), scanning transmission electron microscopy (STEM), scanning electron microscopy (SEM), and BET (Brunauer-Emmett-Teller). the contents of Ag confined in DHSS/SHS were measured by ICP-OES.

Catalytic reduction of nitrobenzene (NB) and 4-nitrophenol (4-NP). An excess amount of $\mathrm{NaBH}_{4}$ was added to $10 \mathrm{~mL}$ of $\mathrm{NB}$ or 4-NP solution $(1.8 \mathrm{mg} / \mathrm{mL}$, unless otherwise noted $)$ in a glass vessel. Thereafter, $0.002 \mathrm{mg} / \mathrm{mL}$ of Ag-DHSS or Ag-SHS and deionized water were added. UV/Vis spectra of the sample were measured on characteristic peak (265 nm for NB, $400 \mathrm{~nm}$ for 4-NP, respectively) in the range of $200-600 \mathrm{~nm}$. To investigate the cyclic stability of catalysts, the used Ag-DHHS catalyst was recycled as follows: For the first run, ten parallel experiments $(0.002$ $\mathrm{mg} / \mathrm{mL}, 10 \mathrm{~mL}$ for each) were performed to obtain the mean conversion ratio of nitrobenzene. For the second cycle, the used Ag-DHHS catalysts were recovered from above ten experiments through filtration, washing with deionized water, and drying at room temperature. All these recovered Ag-DHHS were then re-dispersed in aqueous solution and run for another nine parallel experiments using the same concentration and amount as above, and so on, for each of the eight cyclic experiments. At least three parallel experiments were ensured for the eighth cycle.

\section{Reference}

S1 Chen, M.; Wu, L.; Zhou, S.; You, B.A Method for the Fabrication of Monodisperse Hollow Silica Spheres. Adv. Mater., 2006, 18, 801-806

S2 Jiang, C,; Hara, K.; Fukuoka, A. Low-Temperature Oxidation of Ethylene over Platinum Nanoparticles Supported on Mesoporous Silica. Angew. Chem. Int. Ed. 2013, 52, 6265-6268 

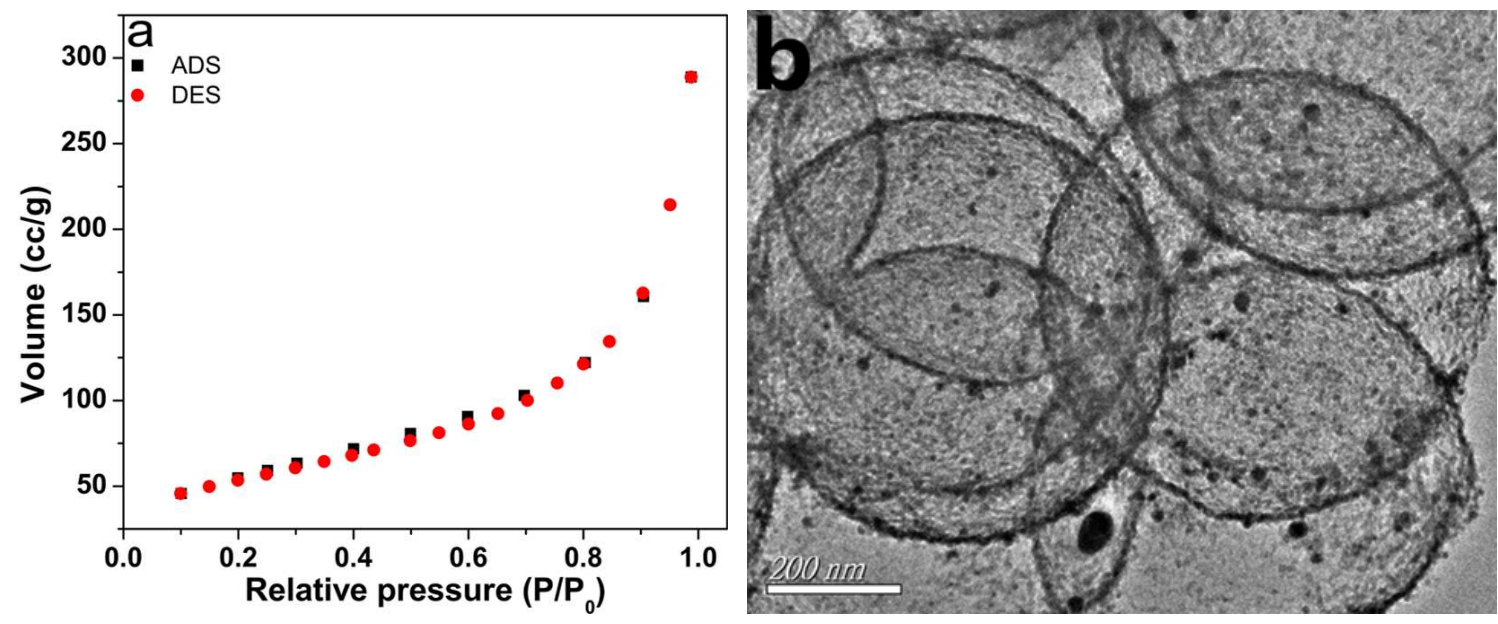

Figure S1. (a) $\mathrm{N}_{2}$ adsorption/desorption isotherms and (b) TEM imageof the typical Ag-SHSS
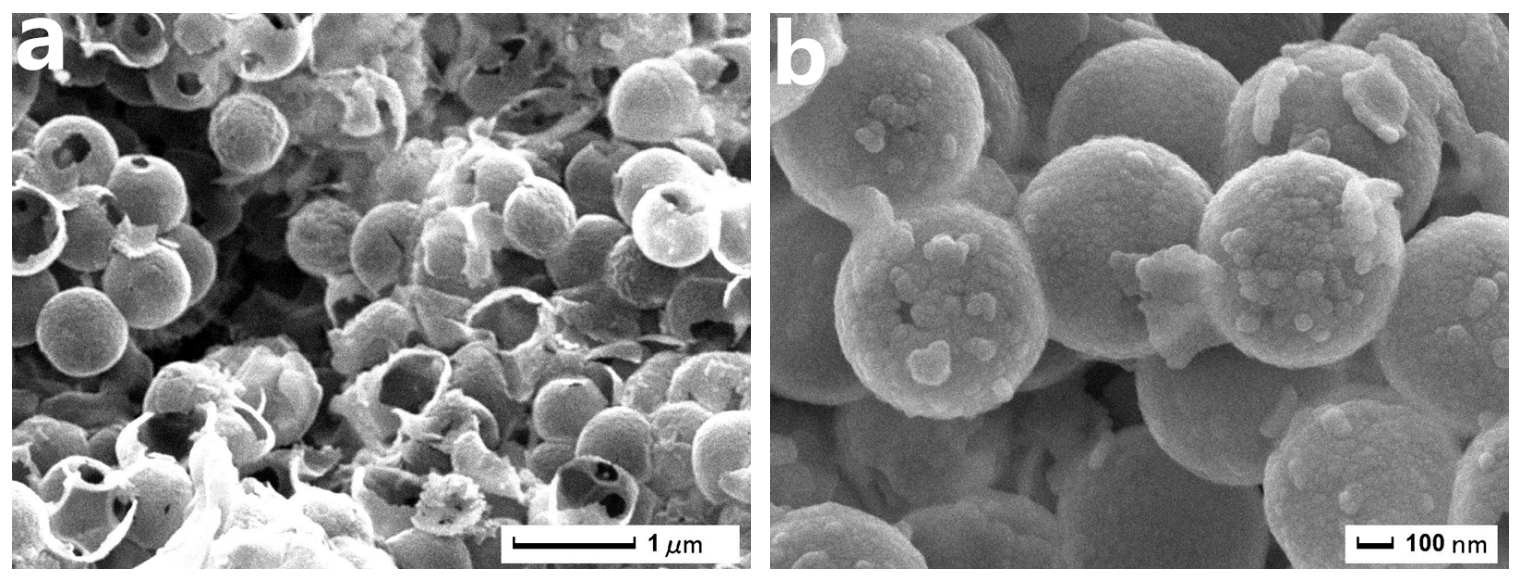

Figure S2. SEM images of the Ag-SHSS (a) and Ag-DHSS (b) after the reaction of NB by $\mathrm{NaBH}_{4}$ 


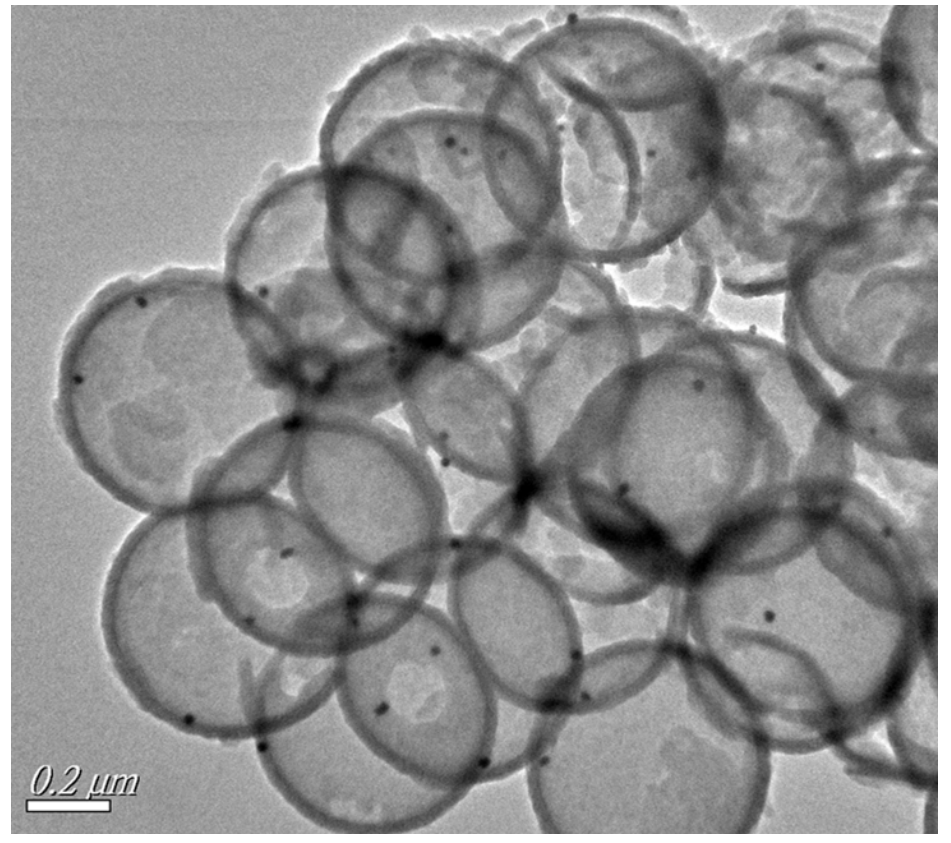

Figure S3. TEM image of Au-DHSS catalyst
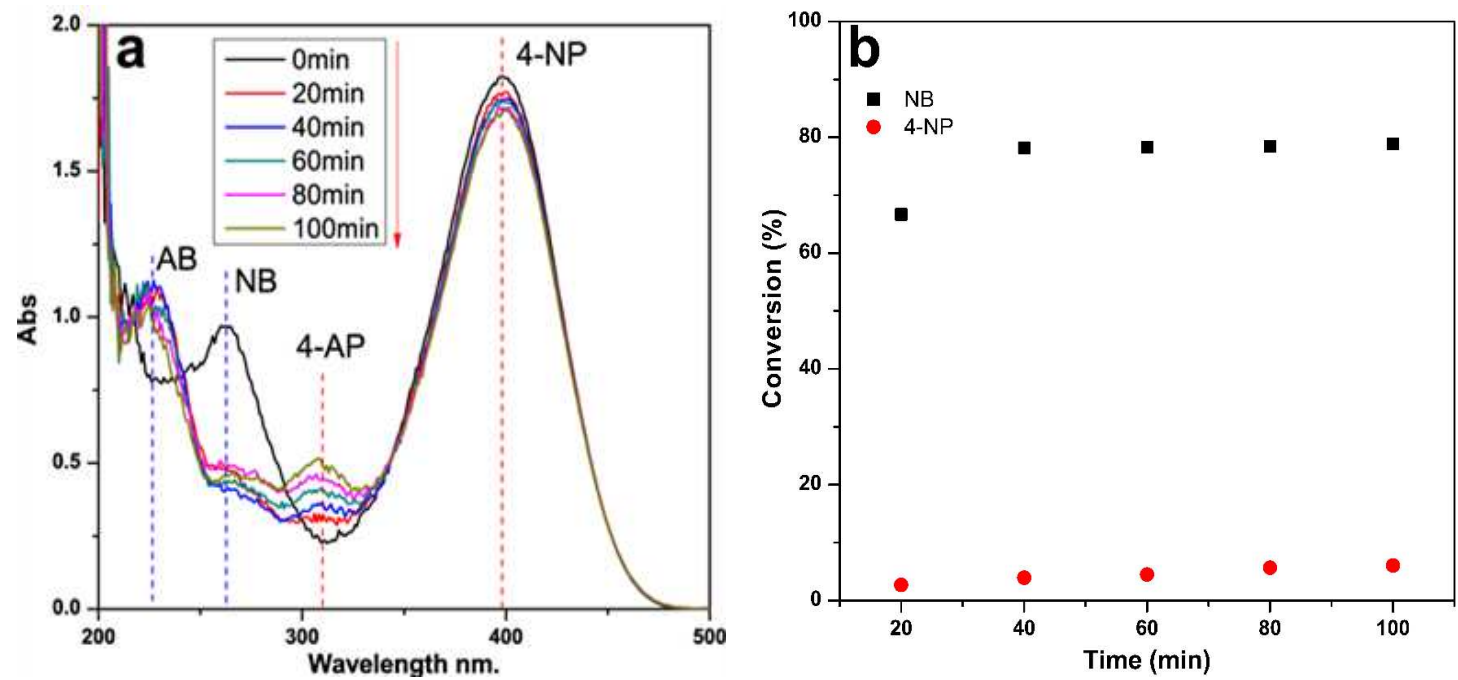

Figure S4. $(a, b)$ The reduced mixture of NB and 4-NP using Au-DHSS as catalyst $\left(20^{\circ} \mathrm{C}, 1.4\right.$ $\mathrm{mg} / \mathrm{mL}$ ). 

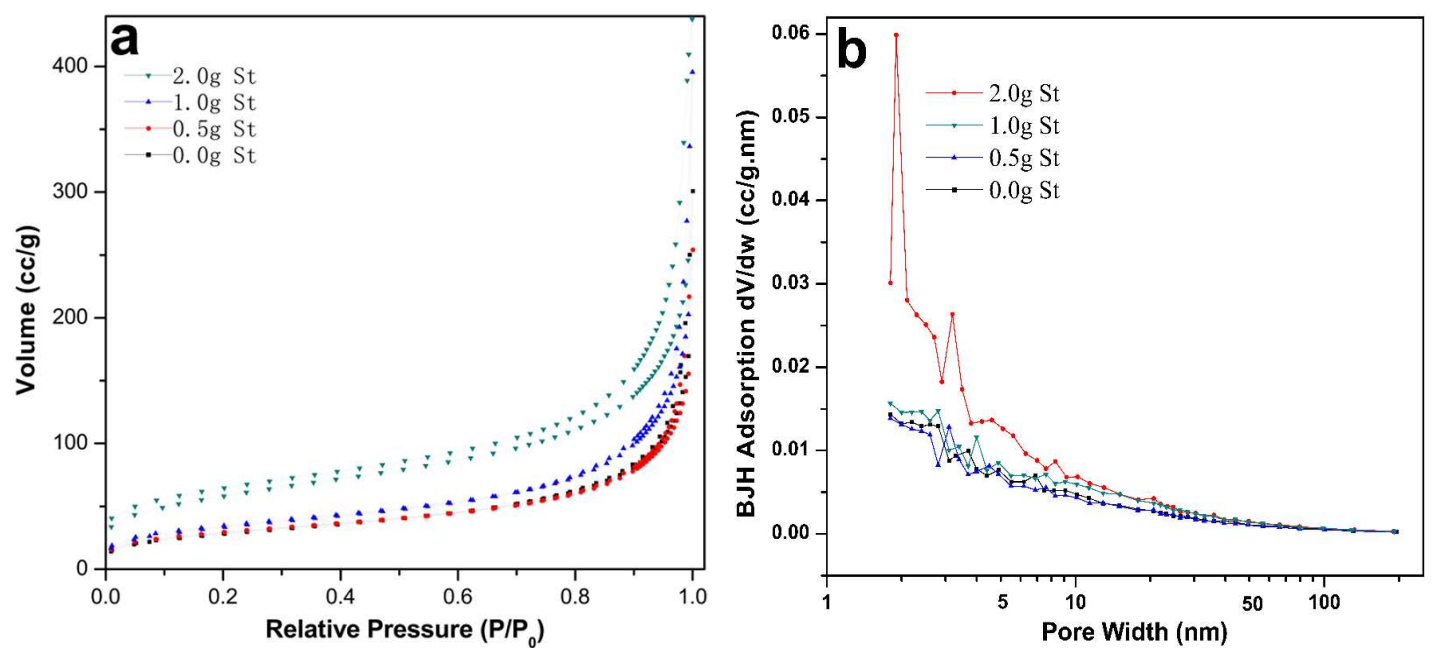

Figure S5. BET isotherms and BJH pore size distributions of Ag-DHSS with different amount of Styrene added (second CPS layer)
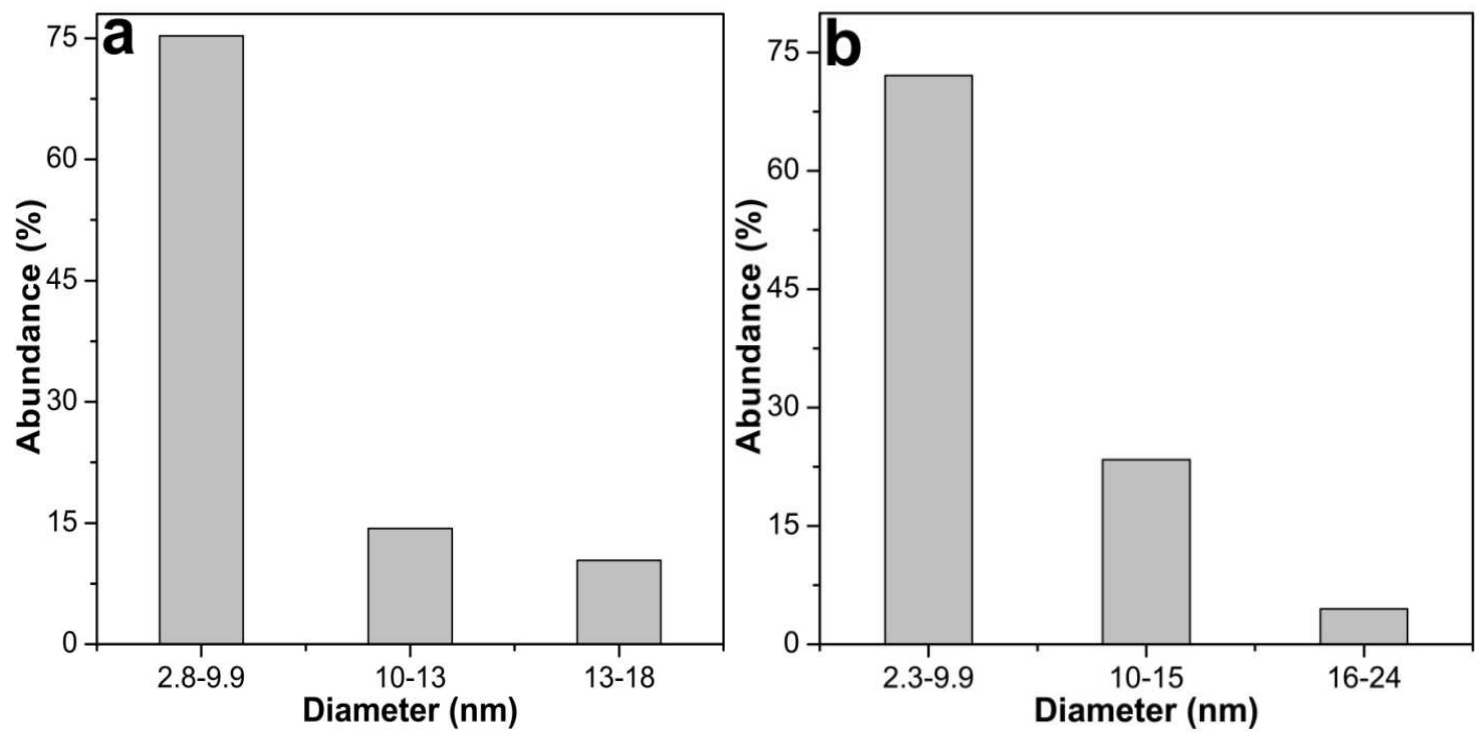

Figure S6. Particle distribution of Ag-DHSS (a) and Ag-SHSS (b) 